\title{
Research Paper \\ Effect of Healthy Lifestyle Educational Programs on Happiness and Life Satisfaction in the Elderly: A Randomized Controlled Trial Study
}

\author{
Mohammad Mohammadi Mehr ${ }^{1,2}{ }^{\circ},{ }^{*}$ Fereshteh Zamani-Alavijeh ${ }^{1} \oplus$, Akbar Hasanzadeh $^{3}$, Tayebeh Fasihi $^{4}$
}

1. Department of Health Education and Health Promotion, School of Health, Isfahan University of Medical Sciences, Isfahan, Iran.

2. Student Research Committee, Isfahan University of Medical Sciences, Isfahan, Iran.

3. Department of Biostatistics and Epidemiology, School of Health, Isfahan University of Medical Sciences, Isfahan, Iran.

4. Social Determinants of Health Research Center, Alborz University of Medical Sciences, Karaj, Iran.

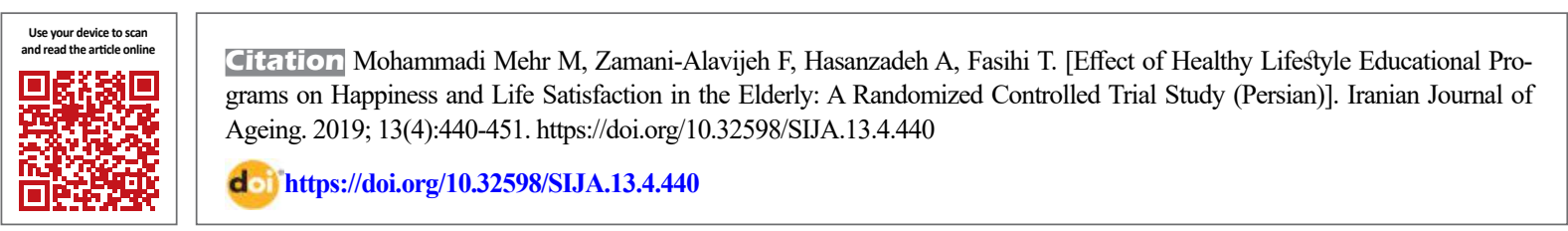

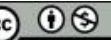

Received: 10 Jun 2018 Accepted: 27 Oct 2018 Available Online: 01 Jan 2019

Keywords: Life satisfaction, Elderly, Training Healthy lifestyle

\section{A B STRACT}

Objectives Like the global population, the Iranian population is aging rapidly. Measures should be taken to maintain aging population healthy and happy. The present study evaluated the effects of active education of healthy lifestyle on happiness and life satisfaction of the elderly people.

Methods \& Materials In this randomized controlled trial, 96 elderly people aged 60-70 were conveniently recruited from a health center in Isfahan Province, Iran. The subjects were randomly allocated to an intervention and a control group. Participants in the intervention group were provided with lifestyle educations in 4 two-hour sessions. Happiness and life satisfaction were assessed before and 2 months after the intervention. Study data were analyzed by SPSS using the Independent samples t-test and Paired t-test, Mann Whitney U test, Wilcoxon signed-rank test, Chi-squared test and Pearson correlation analysis. Results The lifestyle education program significantly improved happiness scores from 36.80 to 43.11 and life satisfaction scores from 12.96 to 16.02 ( $P<0.001)$. Happiness was positively correlated with life satisfaction ( $r=0.719 ; \mathrm{P}<0.001)$.

Conclusion Active participation in healthy lifestyle education programs can improve happiness and life satisfaction among elderly people.

\section{Extended Abstract}

\section{Objectives}

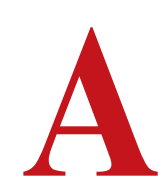

ging is growing rapidly in the world population as well as in Iran [1]. Aging reduces abilities and sometimes leads to a feeling of worthlessness and dependence on others. Such matters decrease satisfaction and happiness in the aged groups [2, 3].
Therefore, there is a need for interventions to keep the elderly healthy and happy $[4,5]$. Several studies investigated happiness and life satisfaction in the Iranian elderly population $[3,6,7]$. However, the impact of education of healthy lifestyle using educational packages of the Iranian Ministry of Health [8] on the happiness and life satisfaction in the elderly remains unknown. Thus, the current study evaluated the effect of these healthy lifestyle educational packages on the happiness and life satisfaction of the elderly in Iran.

\section{* Corresponding Author:}

Fereshteh Zamani-alavijeh, PhD.

Address: Department of Health Education and Health Promotion, School of Health, Isfahan University of Medical Sciences, Isfahan, Iran Tel: +98 (313) 7923292

E-mail: fe.zamani@gmail.com 


\section{Methods \& Materials}

This was a randomized controlled trial study conducted in 2016, on 96 elderlies referring to Comprehensive Health Services Center in Isfahan Province, Iran. The samples were selected using convenience sampling method. The subjects were randomly divided into the trial $(n=48)$ and control $(n=48)$ groups. Inclusion criteria were being 60-70 years old (because of the need for a relative ability to exercise), having consent to participate in the study, having ability to communicate verbally and to exercise according to a physician.

The data collection tool was a 3-part questionnaire; first part surveyed demographic characteristics through 10 items; second part was the Persian version of Life Satisfaction Index-Z (LSI-Z), and the third part was the Persian version of Oxford Happiness Inventory (OHI). The validity and reliability of the Persian versions of these tools have already been determined $[9,11]$. Before conducting the intervention, the questionnaire was completed by the researcher on behalf of samples through interviewing. Then, 3 groups of 16 subjects were formed and presented group training sessions for almost 2 hours per day.

Each educational subject was provided in 2 sessions. The topics of sessions were selected based on the Ministry of Health's educational package on healthy lifestyle in the elderly, focusing on sports and physical activity, proper sleep, marital relationships, stress management, and happy life. Two months after the intervention, all the participants were interviewed again and the post- test questionnaires were completed for them. Collected data were analyzed by Independent t-test, Paired t-test, Pearson correlation coefficient, Wilcoxon signed-rank test, Chi-squared test and Mann-Whitney $U$ test at a significance level of promotion. This study was approved by the Ethics Committee of Isfahan University of Medical Sciences (code: IR.MUI.REC.1395.3.712).

\section{Results}

Of total 96 samples, 48 were in the trial group and 48 in control group. Three samples in the trial group did not attend classes due to personal reasons and withdrew from the study. In the trial group, $66.7 \%$ of samples were female, $71.1 \%$ married, $33.3 \%$ retired, $15.6 \%$ illiterate, and $35.6 \%$ with fair income. In the control group, these values were $52.1 \%, 87.5 \%, 27.1 \%$, $14.6 \%$, and $33.3 \%$, respectively. In terms of these characteristics, no significant differences were between the 2 groups $(\mathrm{P}>0.05)$.

The Independent t-test and Paired t-test results of the pretest mean score of life satisfaction were 12.96 \pm 5.42 in the trial group and $12.2 \pm 4.55$ in control group. Thus, in both groups, life satisfaction was less than half the maximum achievable score (26). Pretest mean scores of happiness in these groups were $36.80 \pm 14.61$ and $37.02 \pm 10.68$, respectively; both less than the average of the maximum score (87). Pretest mean scores of life satisfaction and happiness were not significantly different between the 2 groups $(\mathrm{P}>0.05)$. After the intervention, the subjects' mean score of life satisfaction increased 3.06 units, and the mean score of happiness 6.31 units.

Table 1. Items of Life Satisfaction Questionnaire, which had a significant positive change after intervention, and the percentage of positive

\begin{tabular}{|c|c|c|}
\hline Items & $\mathbf{r}$ & $\mathbf{P}$ \\
\hline As I grow older, things seem better than I thought they would. & 44.4 & $<0.001$ \\
\hline Most things I do are boring or monotonous. & 44.4 & 0.007 \\
\hline This is the dreariest time of my life. & 33.3 & 0.001 \\
\hline The things I do are as interesting to me as they ever were. & 28.8 & 0.001 \\
\hline Compared to other people, I get down in the dumps too often. & 22.2 & 0.006 \\
\hline I have made plans for things I'll be doing a month or a year from now. & 20 & 0.021 \\
\hline Compared to other people of my age, I make a good appearance. & 15 & 0.008 \\
\hline I am just as happy as when I was younger. & 6.6 & 0.025 \\
\hline
\end{tabular}


This increase in both variables was significant $(\mathrm{P}<0.001)$. This change in both variables also caused a significant difference $(\mathrm{P}<0.01)$ between the two groups after the intervention with respect to these variables.

The Pearson correlation coefficient revealed a strong, positive and significant correlation between happiness and life satisfaction. This correlation was observed after the intervention $(\mathrm{r}=0.719, \mathrm{P}<0.001)$. Wilcoxon signed-rank test results indicated that improvement in life satisfaction score was not observed in all items of LSI-Z. It also revealed that in only 8 items out of 13 items (Table 1), participants had a significant difference. In addition, the improvement in these 8 items was not the same and varied from $6.6 \%$ to $44 \%(\mathrm{P}<0.01)$. In OHI, only in 24 items out of 29 (e.g. sense of optimism about the future, sense of control in life, feeling happy, and finding everything amusing and beautiful), participants showed significant improvement after the intervention whose scores ranged between $6.6 \%$ and $37.7 \%$.

\section{Conclusion}

The study results indicate that the active participation of elderly people in healthy lifestyle education programs can increase their happiness and satisfaction with life. Educational programs provided in health centers should involve the elderly in decision making and prevention or health promotion programs. In this case, these interventions will have more satisfactory results. Such interventions should also be continued to have long-lasting results. The high number of items in questionnaires that made the elderly tired, and the lack of time and manpower were the limitations of this study. Group and participatory training and use of reminiscing technique which encourage participation and the acceptance of training by the participants were among the advantages of this study.

\section{Ethical Considerations}

\section{Compliance with ethical guidelines}

This study has been approved by Research deputy of the School of Health (code: 395712), and the Research Ethics Committee of Isfahan University of Medical Sciences (code: IR.MUI.REC.1395.3.712).

\section{Funding}

This study has been approved by Research deputy of the School of Health (code: 395712).

\section{Authors contributions}

Conceptualization, methodology and validation: Fereshteh Zamani and Akbar Hasanzadeh; Investigation and formal analysis, Resources, and draft preparation: Mohammad Mohammadi Mehr; Editing: Tayebeh Fasihi; and Supervision and project administration: Fereshteh Zamani.

\section{Conflict of interest}

The authors declared no conflict of interest.

\section{Acknowledgements}

Authors would like to thank Health educators and colleagues working at the Comprehensive Health Center in Isfahan for their valuable cooperation. 


\title{
تأثير آموزش مبتنى بر سبك زندكّى ساله، بر شادكامى و رضايت از زندكَى در سالمندان: كار آزمايى كنتر لشده تصادفى
}

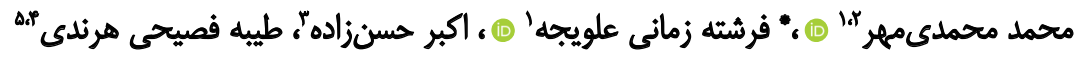 \\ 1- كزوه آموزش بهداشت و ارتقاى سلامت، دانشكده بهداشت، دائشعاه علوم يزشكى اصفهان، اصفهانه ايران.

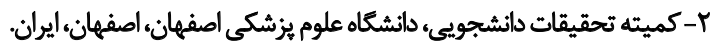

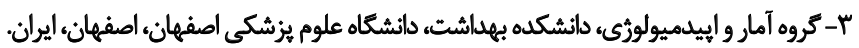

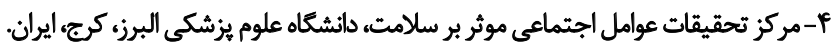

\begin{abstract}
حكب

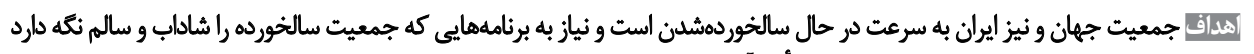

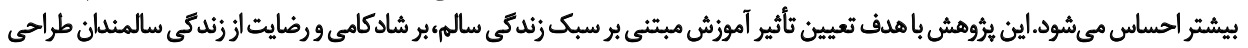
واجرا شده است.

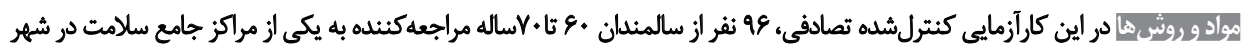

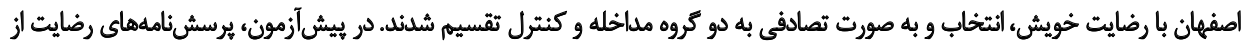

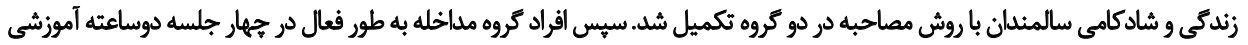

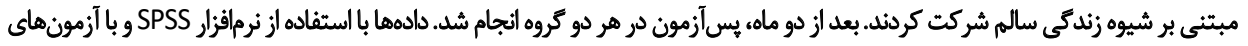

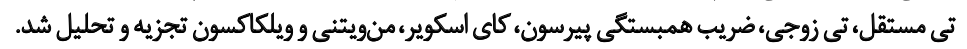

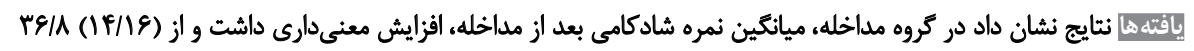

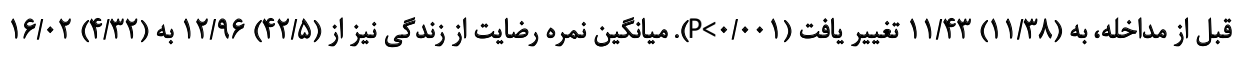

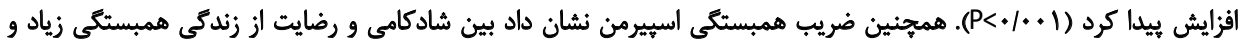

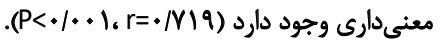

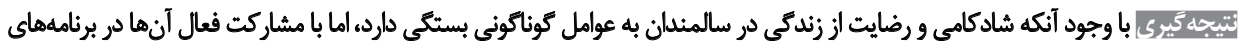

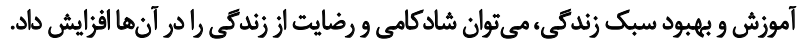

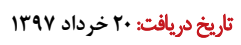

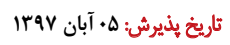

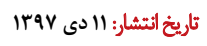

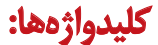

رضايت از زندكي،

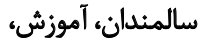
سبك زندكى سالم أموزه

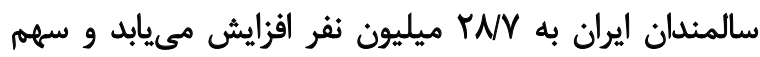

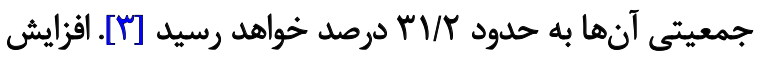

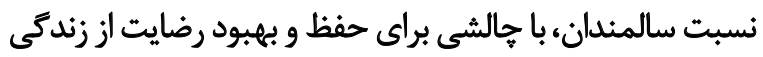

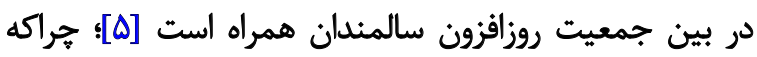

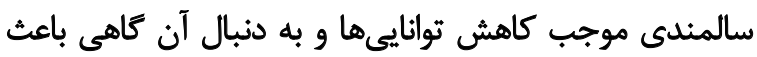

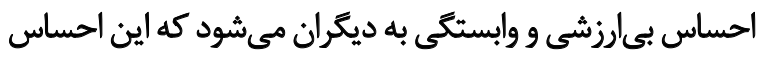

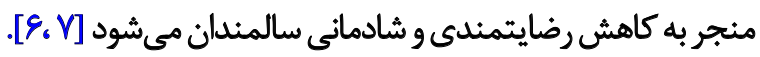

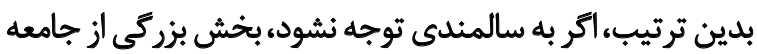

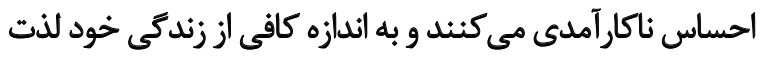

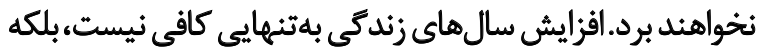

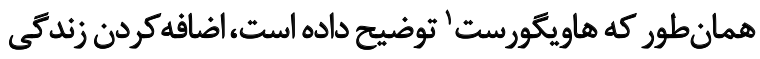

سالمندى، كاهش قواى روانى و جسمى است كه در اثر كذشت

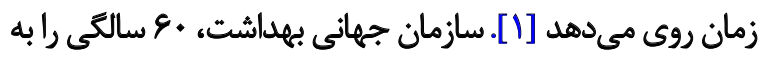

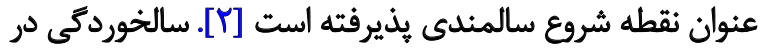

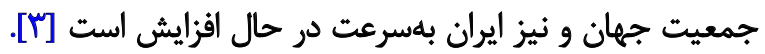

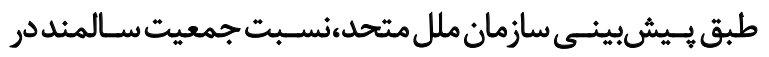

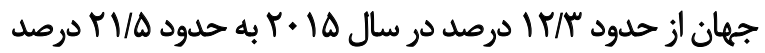

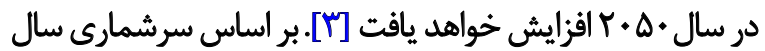

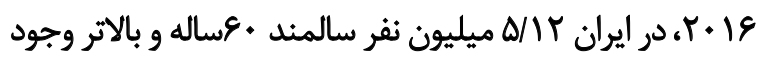

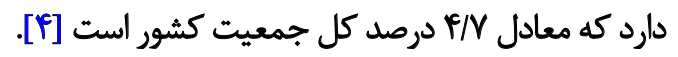

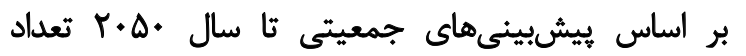

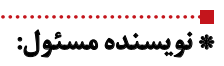

دكتر فرشته زمانى علويجه

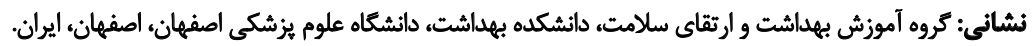
تلفن:

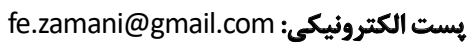




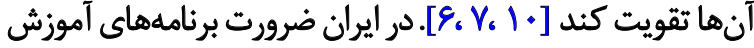

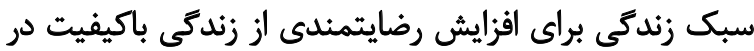

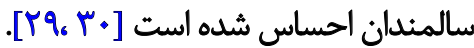

براى آكاهسازى سالمندان در ابعاد مختلف سبك زندكى سالم،

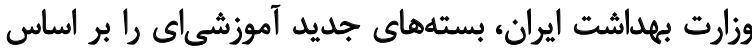

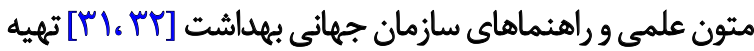

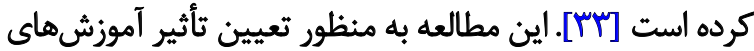

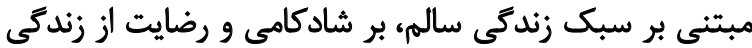

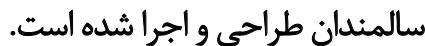

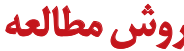

اين يثوهش از نوع كارآزمايى كنترلشده تصادفى ' اسـت.

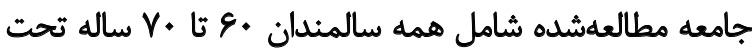

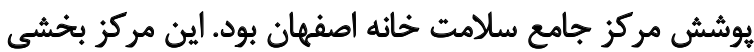

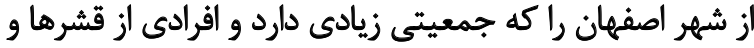

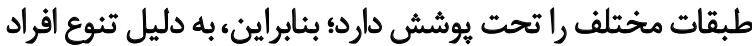

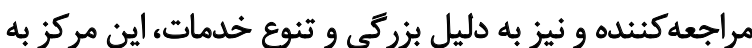

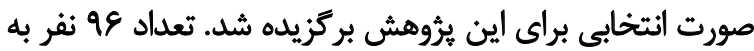

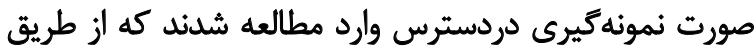

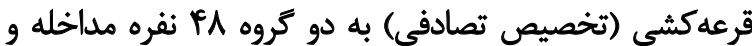
كنترل تقسيم شيند.

معيار هاى ورود به مطالعه: سن •و تا · V سال، سكونت در شهر

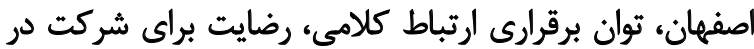

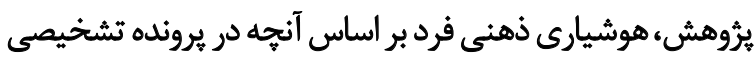

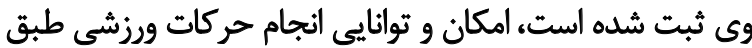

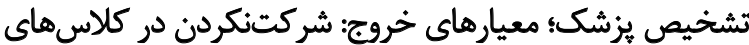

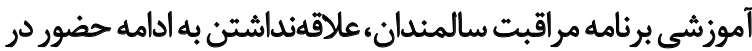
طرح، ازدستدادن توان برقرارى ارتباط يا انجام حركات ورزشى. ابـزار جمـعآورى دادهها يرسشنامه بود كه بـه صـورت

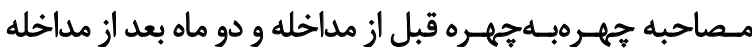

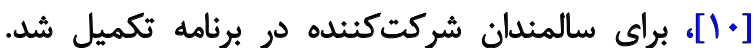

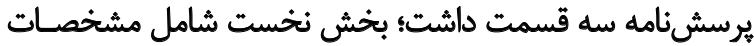

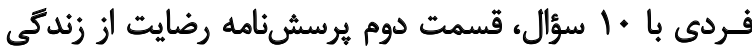
LSI-Z

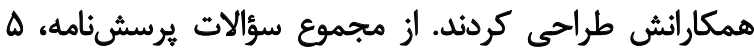

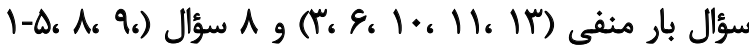

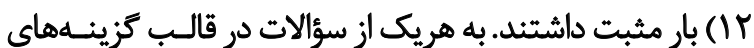

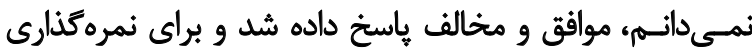

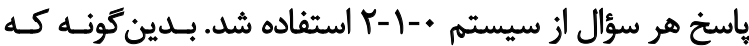

\section{Randomized control trial}

3. Life Satisfaction Index-Z (LSI-Z)

4. Tobin
به سالها و رضايتبخشى زندكى بخش مهمى از تعريف اوليه

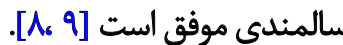

رضايت از زندگى، يك مؤلفه شناختى رفاه ذهنى است كه

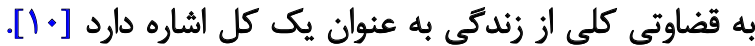

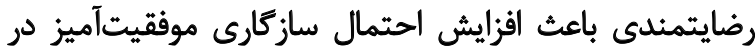

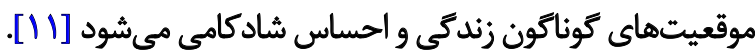

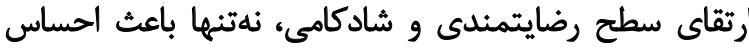

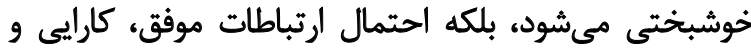

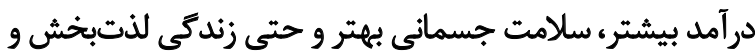

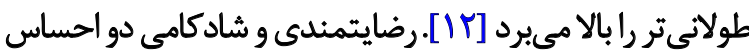

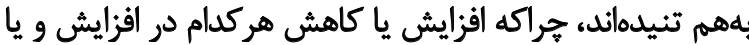

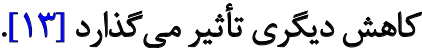

شادكامى، آرامشى فكرى است كه نتايج مطلوبى را براى

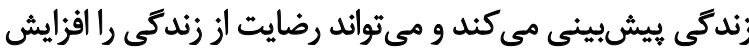

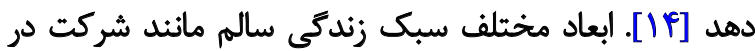

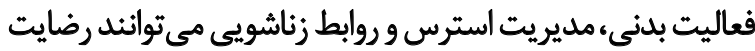

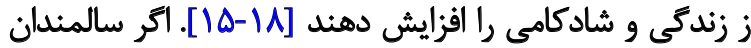

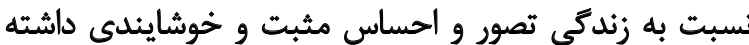

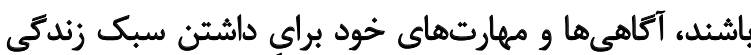

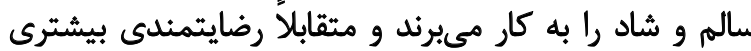

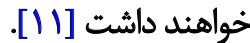

سبك زندگى سالم در سالمندان به مهارت و آكاهى آنان در

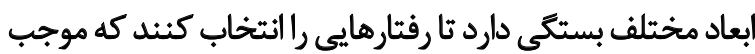

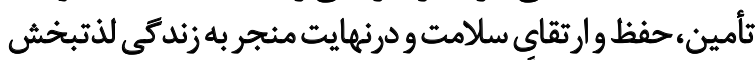

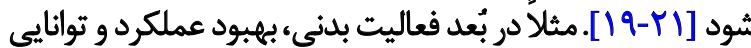

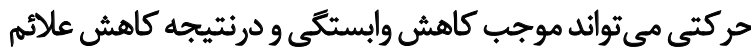

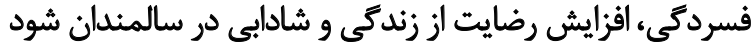

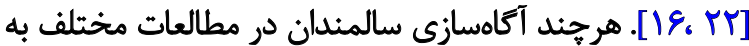

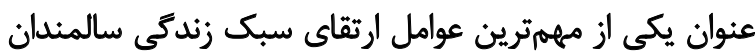

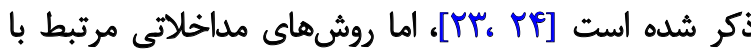

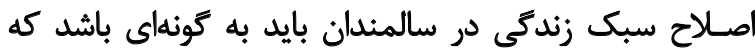

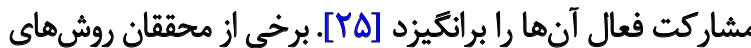

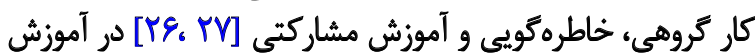

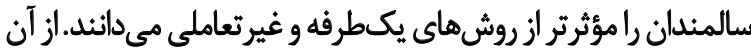

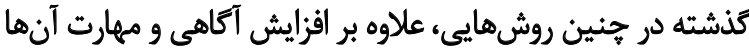

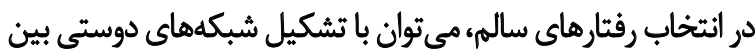

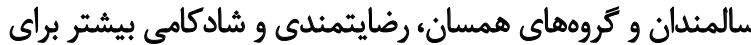

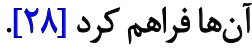

بنابر آنجه توضيح داده شد، براى بهبود رضايتمندى و شادكامى كائى

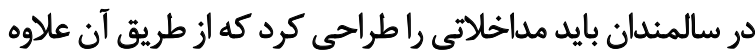

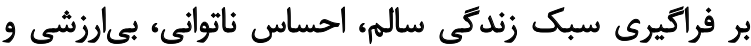

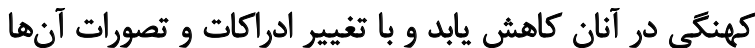

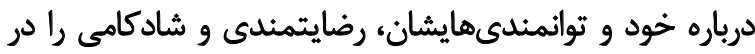


تداوم سبك زندگى سالم به وجود آورد و افراد، متقاعد به انجام

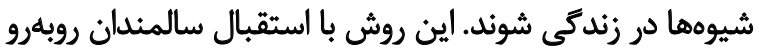

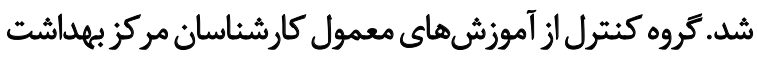
بهرهمند شدند و مداخله ديكرى دريافت نكرئ مكردئد.

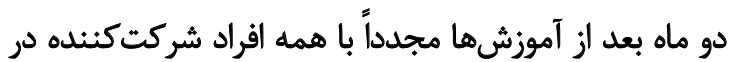

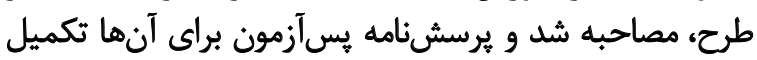

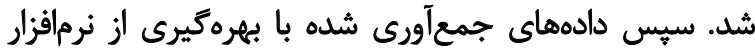

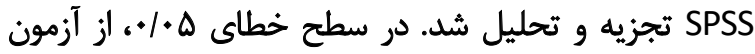

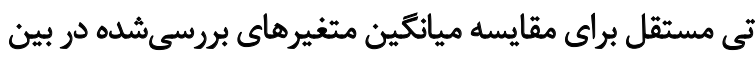

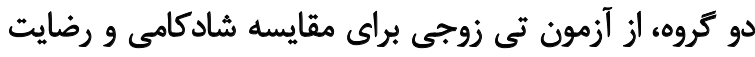

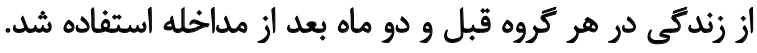

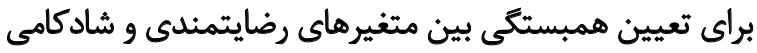

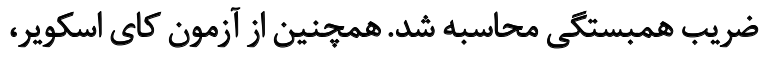

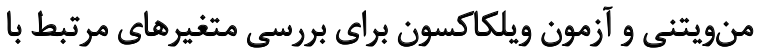

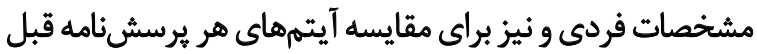

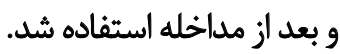

يافتهها

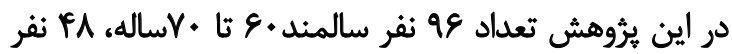

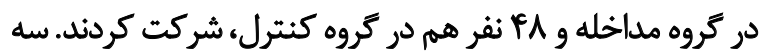

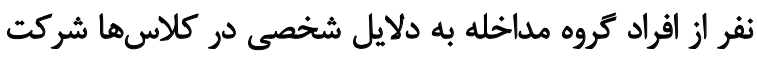

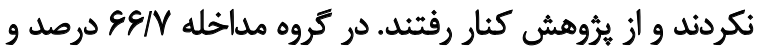

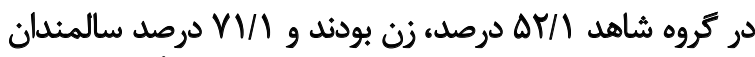

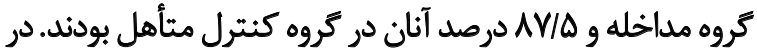

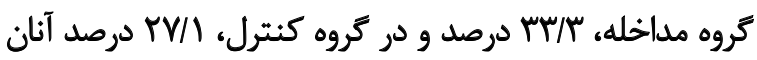

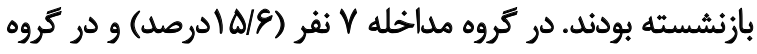

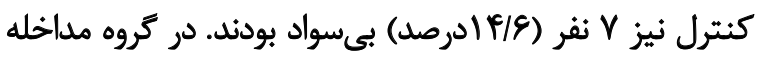

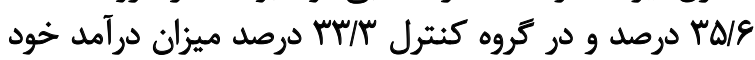

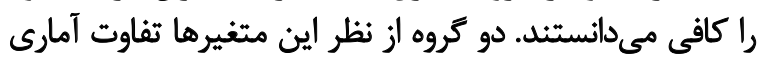

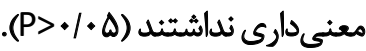

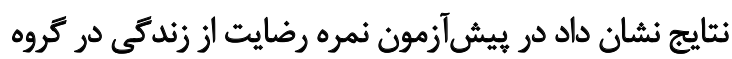
مداخله

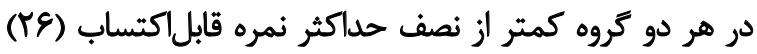

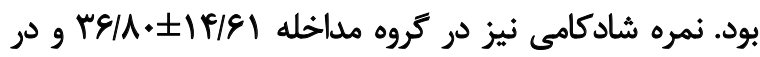

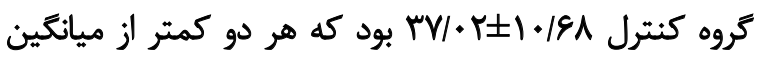

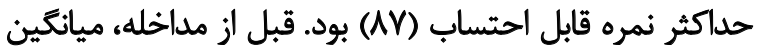

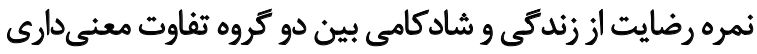

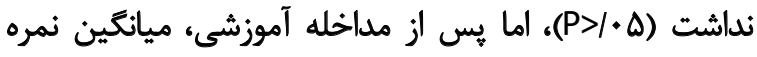

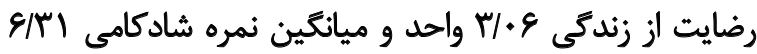

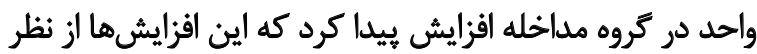

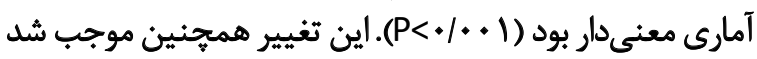

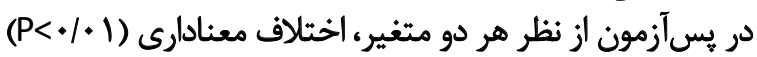
بين دو گروه به وجود بيايد (جدول شماره لمار ).

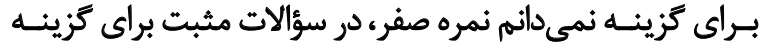

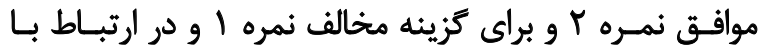

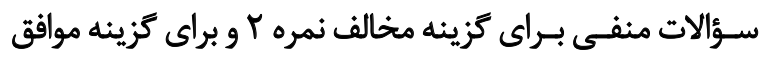

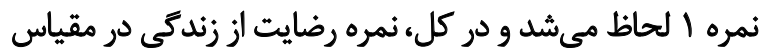
صفر تا ب تعيين شد. محققان طراح، نمرات باك،

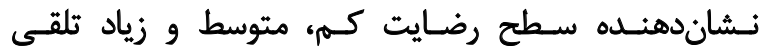

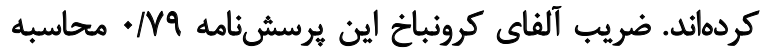

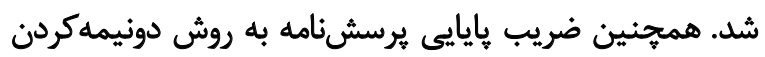

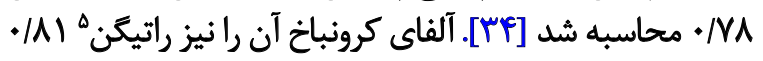

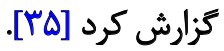

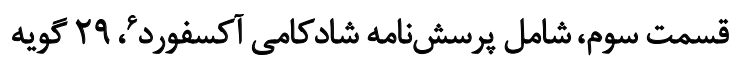

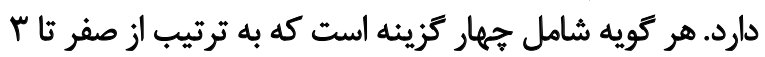

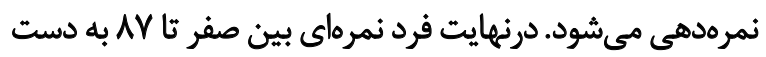

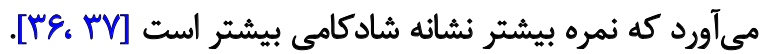

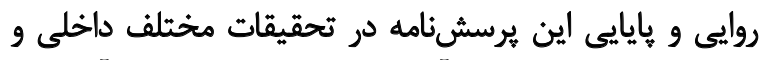

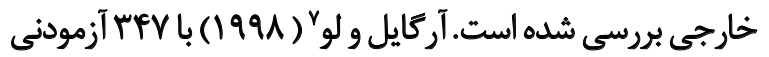

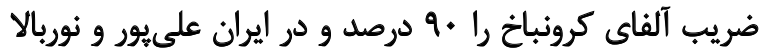

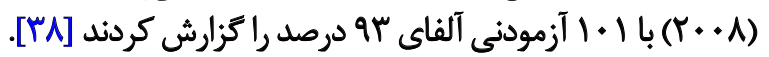

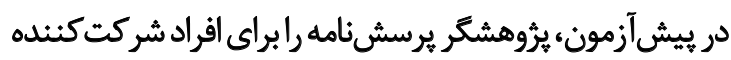

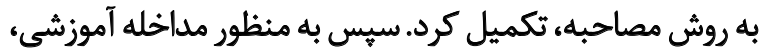

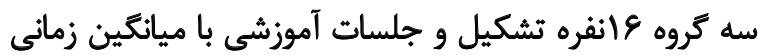

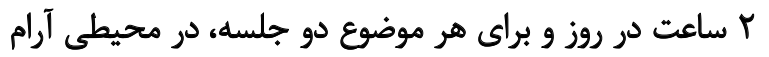

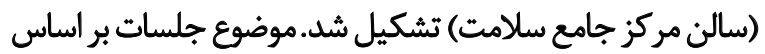

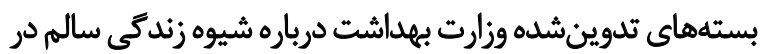

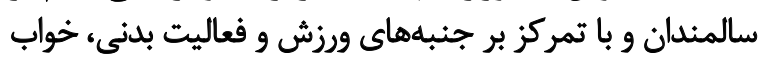

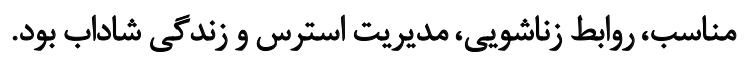
موضوع هر جلسه به اين ترثيب انتخاب شد: جلسه اول، اهميت

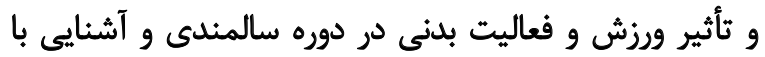

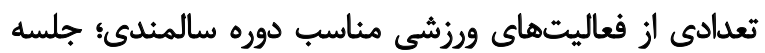

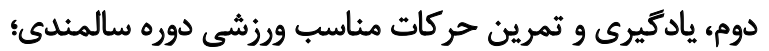

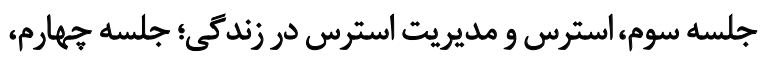

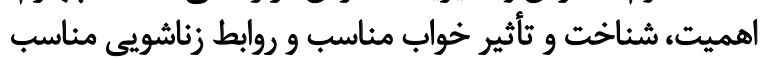
در دوره سالمينلي. كلاسها يك روز در ميان و درمجموع باس جار جلسه آموزشى

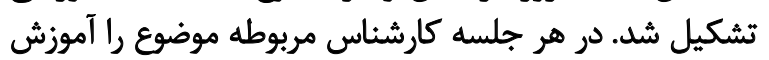

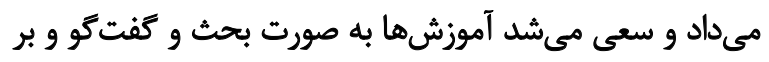

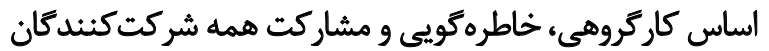

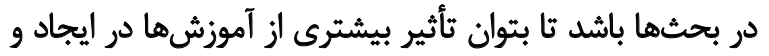

5. Ratigan

6. Oxford Happiness Inventory (OHI)

7.Alipour, Agah Heris 
جدول ا. نمره ميانكين و انحراف معيار رضايت از زندكى و شادكامى قبل و بعد از مداخله در دو كروه

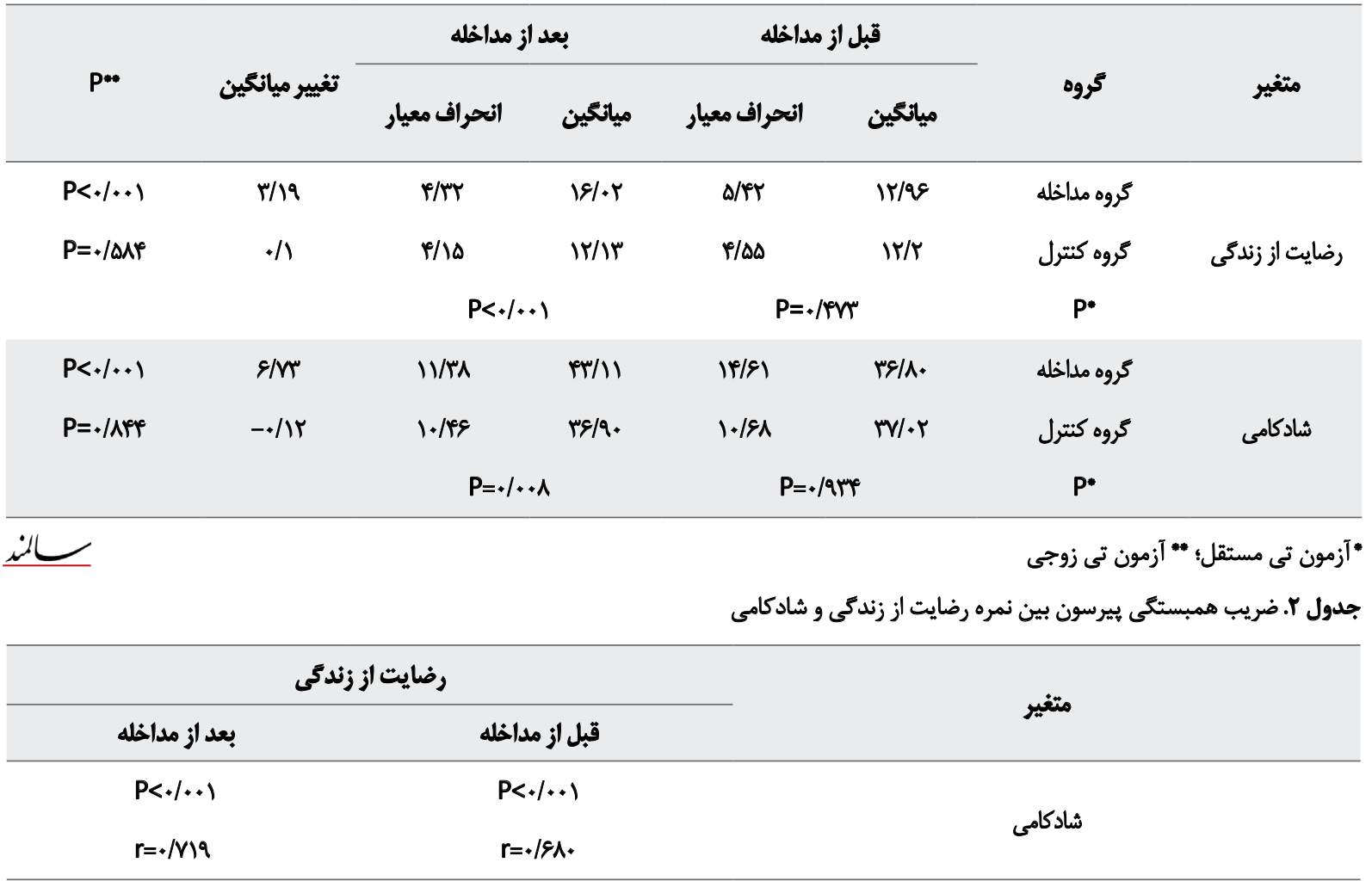

少

درصد متفاوت بوده است (1 (P< (P) (جدول شماره س). در نمره

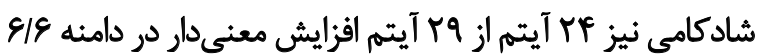

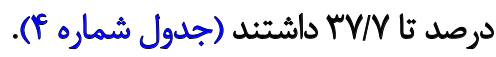

$\stackrel{\leftrightarrow}{\leftrightarrow}$

در اين مطالعه آموزش سبك زندگى سالم در افزايش سطح

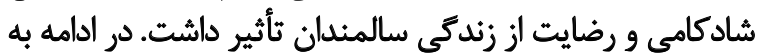

ضريب همبستكى بيرسون نشان داد بين نمره شادكامى و رضايت

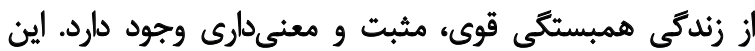

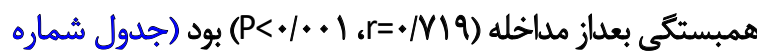

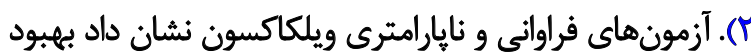

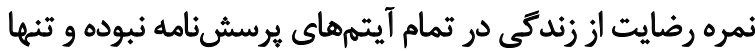

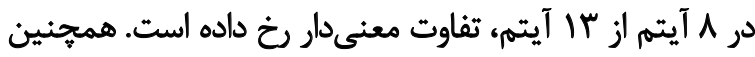

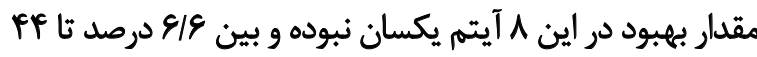

جدول ب. آيتمهاي برسشّنامه رضايت از زندكى كه بعد از مداخله تغيير مثبت معنى دار داشتياند و درصد مثبتشدن آنها

\begin{tabular}{|c|c|c|}
\hline $\mathbf{P}$ & درصد مثبتشدن & 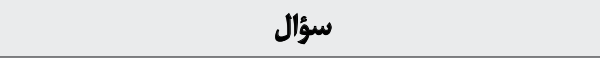 \\
\hline$<\cdot 1 \cdot .+1$ & $p+/ s$ & مطلوببودن روند تعيير شرايط زندكى در دوران سالمندى \\
\hline $.1 . . v$ & $\mu t / f$ & خسته كتنده يا يكنواختبودن بيشتر كارها \\
\hline$+4+1$ & $\pi / r$ & روزهاى سالمندى، ملالتبارثتين روزهاى زندكىبودن \\
\hline $.1+1$ & TNA & جالببودن كارهاى روزمره هانثد كنششته \\
\hline $.1 . .8$ & $r r / r$ & شانس كمتر داشتن در مقايسه با ديكران \\
\hline.$+r$ & r. & برنامهريزى داشتن براى كارهاى آينده \\
\hline$\cdots 1$ & 10 & احساس غُلبه بر شكستهاي زيادى در زندكى نسبت به ديكران \\
\hline .1 .40 & $9 / 8$ & شادبودن به اندازه زمان جواني \\
\hline
\end{tabular}


جدول P. آيتمهاى برسشثنامه شادكامي كه بعد از مداخله تغييرمثبت معنى دار داشتهاند و درصد مثبت شدن آنها

\begin{tabular}{|c|c|c|}
\hline $\mathbf{P}$ & درصد مثبتشدن & 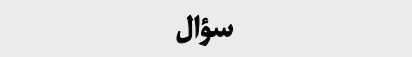 \\
\hline$<\cdot 1 \cdot . \cdot 1$ & WN & رفع خُستكى بعد از خواب \\
\hline$<\cdot 1 \cdot \bullet 1$ & rNA & خوش خيني به آينده \\
\hline$<\cdot 1 \cdot \bullet 1$ & refe & اجساس كثترل بر زندكى \\
\hline$<+/ *+$ & $r e / f$ & احساس شادى \\
\hline$=+1 \cdot+1$ & $r \varphi / p$ & قشنك و زيباديلن بسيارى أز جيزهاى دنيا \\
\hline$<\bullet / \cdot+1$ & $\pi / T$ & هطلوببودن بسيارى از جنبههاي زندكى \\
\hline$<\bullet / . \bullet 1$ & $M / T$ & جالبديلن بسيارى از جيزها \\
\hline$+\infty 1$ & $r M / r$ & احساس سلامتى و تندرستى \\
\hline $.1+.1$ & r. & 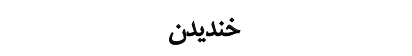 \\
\hline $.1 .+1$ & r. & دوستداشتن زئدىى \\
\hline .1 .01 & $10 / 0$ & هدفمندبونن زندكى \\
\hline$\bullet /+\infty$ & I & انجامدادن كارهايى كه دوست داثتلهام \\
\hline $.1+11$ & $1 \% / r$ & احساس داشتن توان و نيرو \\
\hline.$/ .18$ & $m / r$ & الحساس هوشياربودن \\
\hline $.1 . .1$ & $M /$ & حالت شور و شوق داشتن \\
\hline.$/+\circ$ & $11 /$ & احساس تعهل و فعاليت \\
\hline.$/+r$ & $11 / 1$ & ت تنظيم وقت \\
\hline$\bullet / .+r$ & $11 /$ & احساس صميميت با ديكران \\
\hline $.1 \cdot 0$ & 111 & خوببودن دئيا \\
\hline.$/ \circ 1 f$ & $M / 1$ & خاطرههاى خوش كنشته \\
\hline.$/ .14$ & NA & دشواربودن شروع كارها \\
\hline $.1 . Y 0$ & 818 & علاقه به ديكران \\
\hline $.1 . m$ & sig & از بودن با ديكران لذتبردن \\
\hline .1 .48 & $9 / 8$ & احساس رضايت از خود \\
\hline
\end{tabular}


فرهنكى، اجتماعى و محيطى دليل اختلاف در آمار ذكرشده

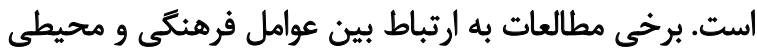

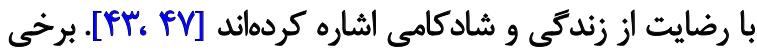

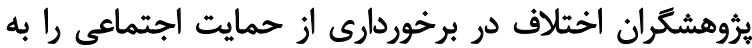

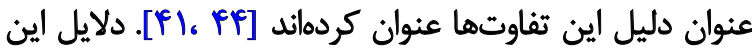

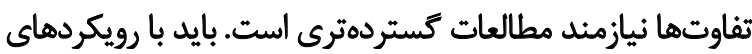

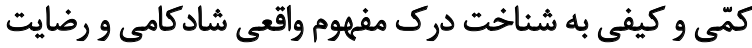

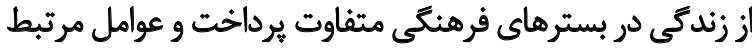

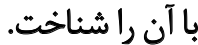

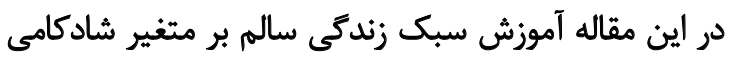

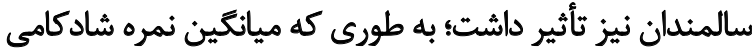

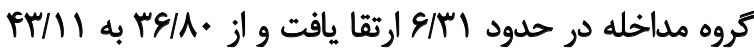

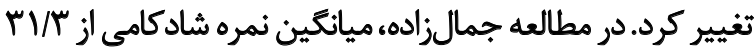

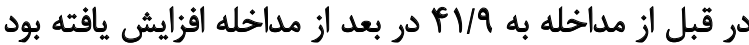

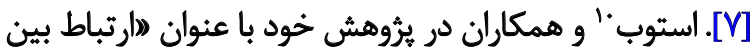

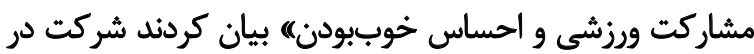

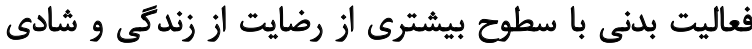

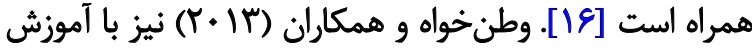

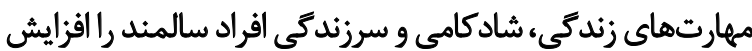

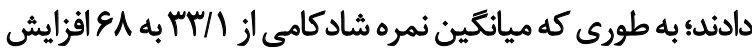

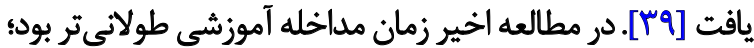

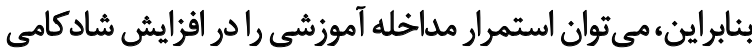

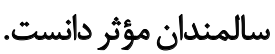

\section{نتيجلكَيرى نههايى}

اين يثوهش تأييد كرد كه مى توان با مشاركت فعال سالمندان

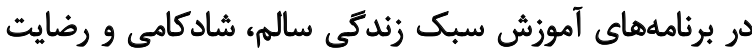

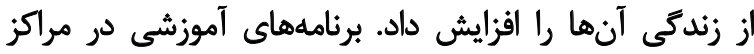

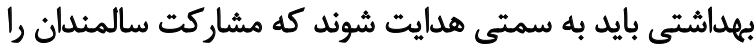

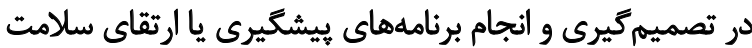

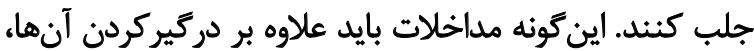

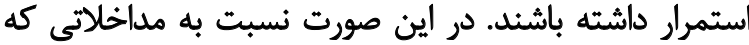

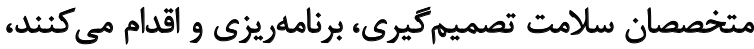
نتايج رضايتبخشترى دارند.

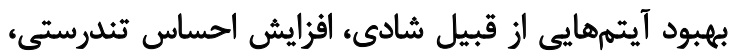

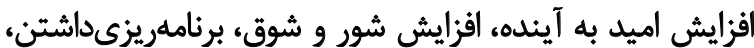

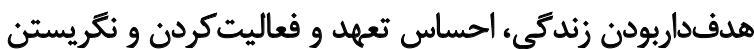

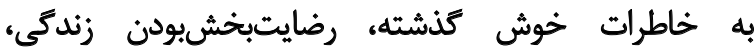

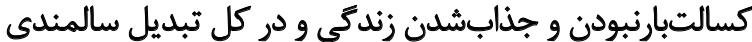

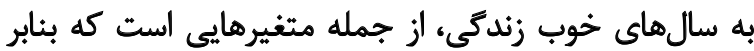

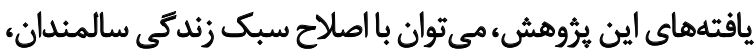

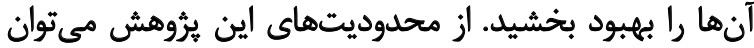

مقايسه و بحث بيرامون نتايج اين مطالعه با نتايج ساير مطالعات

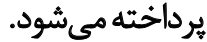

نتايج اوليه اين مطالعه همسو با برخى مطالعات قبلى نشان داد داد

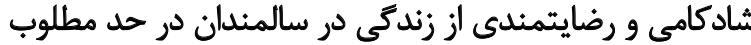

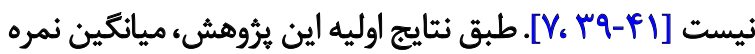

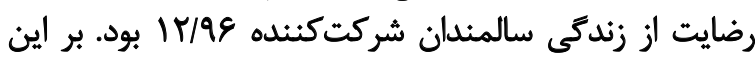

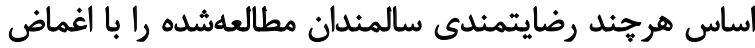

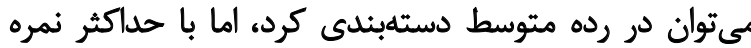

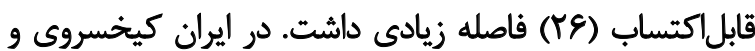

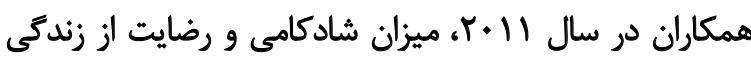

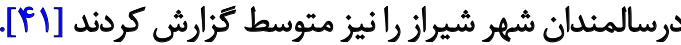

V. اوانس ^ با استفاده از ابزار مشابه، ميانكين رضايتمندئ

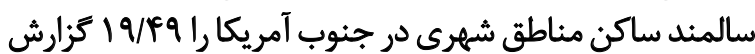

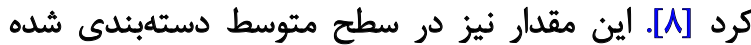

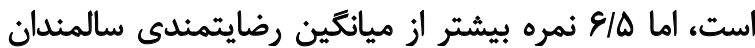

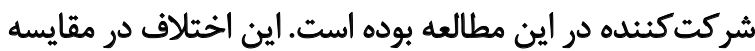

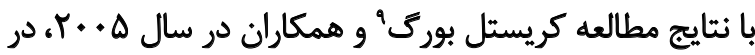

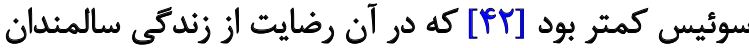

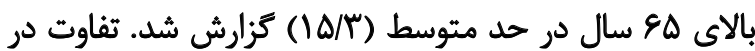

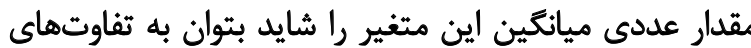

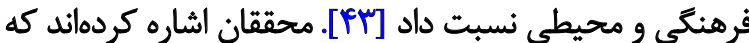

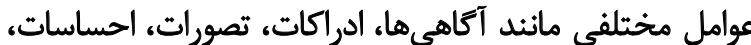

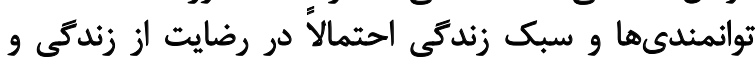

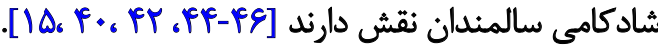

در اين مطالعه، نتايج نشان داد در سالمندانى كه در معرض

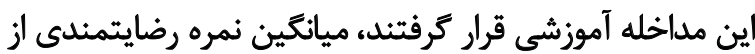

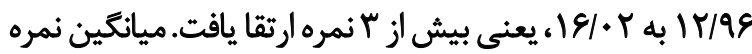

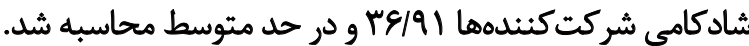

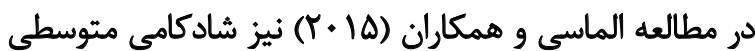

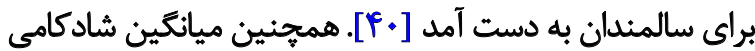

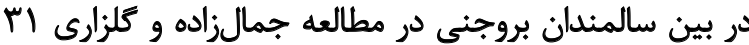

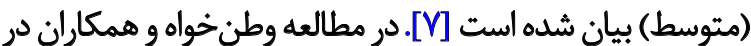

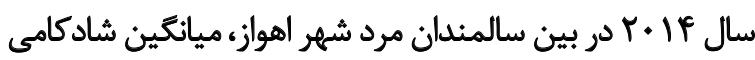
بر اساس برسشنامه شادكامى آكسفورد، آهآ (متوسط) بيان

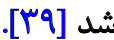

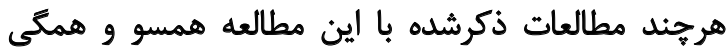

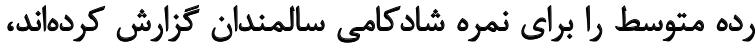

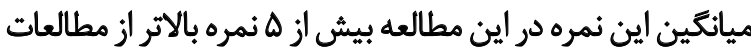

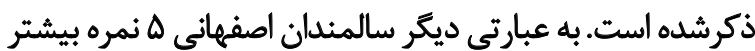

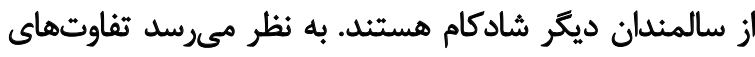


به زيادبودن آيتمهاى يرسشنامهها كه باعث خستكى سالمندان

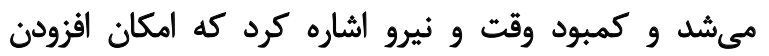

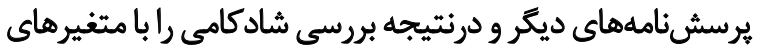

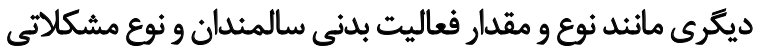

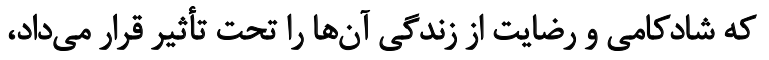

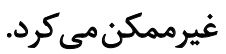

وبيشنهاد مى شود براي آموزش سبك زندكَ از آموزش يكسطرفه

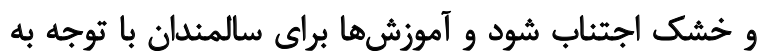

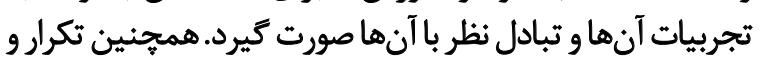

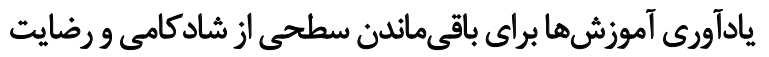

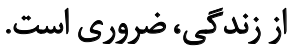

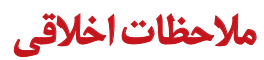
بيروى أز اصول اخلاق يثوهش

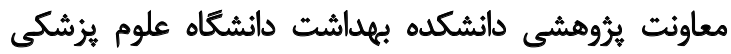

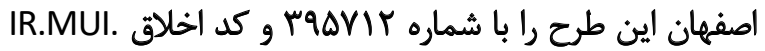
REC.1395.3.712

$$
\text { مامى مالى }
$$

اين مقالهازيايان نامهمحمدمحمدى مهردرد دوره كارشناسىارشد

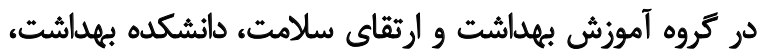

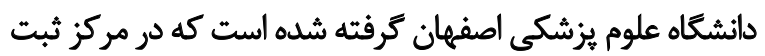

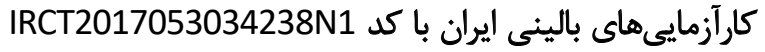

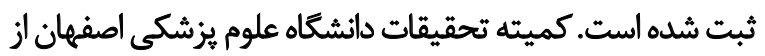
اين يُرؤوش حمايت مالى كرده است.

$$
\text { مشاركت ثويسئدمان }
$$

مفهومسازى، روششناسى، اعتبارسنجى: فرشته زمانى و اكبر

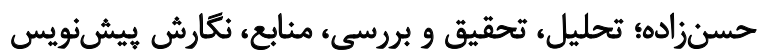

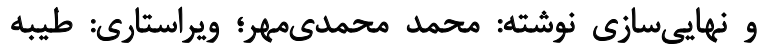
فصيحى؛ نظارت، مديريت يروزه: فرشته زمانى.

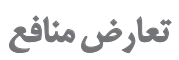
بنابر اظهار نويسندكان، اين مقاله تعارض منافع ندارد.

$$
\text { تشيكر و قدوراني }
$$

از آموزشكُران بهداشتى و همكاران شاغل در مركز جامع سلامت

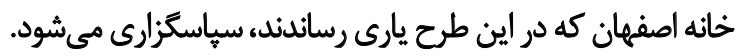




\section{References}

[1] Masnavi A. Causes of the deposit to the sanitarium ageing. The Faculty of Medical science. Philadelphia: Saunders; 1998.

[2] World Health Organization. Aging and life course. Geneva: World Health Organization.

[3] World Health Organization. World population ageing. Geneva: World Health Organization.

[4] Statistical Centre of Iran. [The census of 2016 (Persian)]. Tehran: Statistical Centre of Iran; 2017.

[5] Kooshair H, Yahaya N, Hamid TA, Abu Samah A. Gender as a moderator of the relation among social support functions and life satisfaction in older Malaysians. Journal of Women \& Aging. 2014; 26(1):22-38. [DOI:10.1080/08952841.2014.858550] [PMID]

[6] Mollaoğlu M, Tuncay FÖ, Fertelli TK. Mobility disability and life satisfaction in elderly people. Archives of Gerontology and Geriatrics. 2010; 51(3):e115-9. [DOI:10.1016/j.archger.2010.02.013] [PMID]

[7] Jamalzadeh R, Golzari M. [The effectiveness of hope therapy on increasing happiness and life satisfaction among elderly women residing in nursing home of Boruje (Persian)]. Women and Community of Journal. 2014; 5(18):48-31.

[8] Evans RJ. A comparison of rural and urban older adults in Iowa on specific markers of successful aging. Journal of Gerontological Social Work. 2009; 52(4):423-38. [DOI:10.1080/01634370802609197] [PMID]

[9] Jin B. Social psychological determinants of life satisfaction in older adults [PhD. dissertation]. Bloomington: Indiana University Bloomington; 2001.

[10] Haybron D. Life satisfaction, ethical reflection, and the science of happiness. Journal of Happiness Studies. 2007; 8(1):99-138. [DOI:10.1007/s10902-006-9006-5]

[11] Erdogan B, Bauer TN, Truxillo DM, Mansfield LR. Whistle while you work: A review of the life satisfaction literature. Journal of Management. 2012; 38(4):1038-83. [DOI:10.1177/0149206311429379]

[12] Kushlev K, Heintzelman SJ, Lutes LD, Wirtz D, Oishi S, Diener E. Enhance: Design and rationale of a randomized controlled trial for promoting enduring happiness \& well-being. Contemporary Clinical Trials. 2017; 52:62-74. [DOI:10.1016/j. cct.2016.11.003] [PMID]

[13] Decancq K, Michiels A. Measuring successful aging with respect for preferences of older persons. The Journals of Gerontology, Series B: Psychological Sciences and Social Sciences. 74(2):364372. [DOI: $10.1093 /$ geronb/gbx060]

[14] Andersson P. Happiness and health: Well-being among the self-employed. The Journal of Socio-Economics. 2008; 37(1):21336. [DOI:10.1016/j.socec.2007.03.003]

[15] Kawamoto R, Doi T, Yamada A, Okayama M, Tsuruoka K, Satho $\mathrm{M}$, et al. Happiness and background factors in community-dwelling older persons. Japanese Journal of Geriatrics. 1999; 36(12):861-7. [DOI:10.3143/geriatrics.36.861]

[16] Stubbe JH, De Moor MH, Boomsma DI, de Geus EJ. The association between exercise participation and well-being: A co-twin study. Preventive Medicine. 2007; 44(2):148-52.[DOI:10.1016/j. ypmed.2006.09.002] [PMID]
[17] Shirbeigi M, Esmaili Z, Sarmadi MR, Moradi A. [Study and comparing 3 groups of active, passive, and ecotourist old age people on their mental health and happiness living in Illam City, Iran (Persian)]. Iranian Journal of Ageing. 2016; 10(4):40-9.

[18] Rafiee S, Toozandehjani H, Ahooei MR. [Relationship of lifestyle and social support with marital satisfaction of elderly population (Persian)]. Iranian Journal of Ageing. 2016; 11(2):226-33.

[19] Cockerham WC. The social determinants of the decline of life expectancy in Russia and Eastern Europe: A lifestyle explanation. Journal of Health and Social Behavior. 1997; 38(2):117-30. [PMID]

[20] Babak A, DavariS, Aghdak P, Pirhaji O. [Assessment of healthy lifestyle among elderly in Isfahan, Iran (Persian)]. Journal of Isfahan Medical School. 2011; 29(149):53-59.

[21] Najimi.A, Moazemi.A. [Healthy lifestyle of the elderly: A cross-sectional study (Persian)]. Health System Research. 2012; 8(4):581-7.

[22] Garatachea N, Molinero O, Martínez García R, Jimenez Jimenez R, Gonzalez Gallego J, Marquez S. Feelings of well being in elderly people: Relationship to physical activity and physical function. Archives of Gerontology and Geriatrics. 2009; 48(3):306-12. [DOI:10.1016/j.archger.2008.02.010] [PMID]

[23] Sharifirad G, Najimi A, Hassanzadeh A, Azadbakht L. Ap plication of BASNEF educational model for nutritional education among elderly patients with type 2 diabetes: Improving the glycemic control. Journal of Research in Medical Sciences. 2011; 16(9):1149-58. [PMCID]

[24] Najimi A, Azadbakht L, Hassanzadeh A, Sharifirad G. [The effect of nutrition education on risk factors of cardiovascular diseases in elderly patients with type 2 diabetes: A randomized controlled trial (Persian)]. Iranian Journal of Endocrinology and Metabolism. 2013; 5(2):157-62.

[25] Haber D. Health Promotion and Aging. New York: Springer Publishing Company; 2010.

[26] Yousefi Z, Sharifi K, Tagharrobi Z, Akbari H. The effect of narrative reminiscence on happiness of elderly women. Iranian Red Crescent Medical Journal. 2015; 17(11):e19612. [DOI:10.5812/ ircmj.19612] [PMID] [PMCID]

[27] Jahanbin I, Mohammadnejad S, Sharif F. The effect of group reminiscence on the cognitive status of elderly people supported by Ilam welfare organization in 2013: A randomized controlled clinical trial. International Journal of Community Based Nursing and Midwifery. 2014; 2(4):231-9. [PMID] [PMCID]

[28] Tomini F, Tomini SM, Groot W. Understanding the value of social networks in life satisfaction of elderly people: A comparative study of 16 European countries using SHARE data. BMC Geriatrics. 2016; 16:203. [DOI:10.1186/S12877-016-0362-7] [PMCID]

[29] Rasol Zadeh M, Safavi Farroki S, Amozadeh Khalili M, Pahlavanian A. [Study of interest to individual reside in elderly nursing home (Persian)]. Academic Journal of Semnan University of Medical Sciences. 2008; 10(1):42-8

[30] Mahmudi G, Sanati T. [Evaluation of life style in the elderly (Persian)]. Health of Family. 2013; 1(3):45-50

[31] Word Health Organization. In role of physical activity in healthy ageing. Geneva: Word Health Organization; 1998. 
[32] Word Health Organization. Age-friendly primary health care centres toolkit. Geneva: World Health Organization; 2008.

[33] Majd M, Azimi M. [Healthy lifestyle in the elderly: Nutrition and sport (Persian)]. Tehran: Ministry of Health of Iran; 2003.

[34] Tagharrobi Z, Tagharrobi L, Sharifi K, Sooki Z. [Psychometric evaluation of the Life Satisfaction Index-Z (LSI-Z) in an Iranian elderly sample (Persian)]. Payesh. 2011; 10(1):5-13.

[35] Ratigan A, Kritz Silverstein D, Barrett Connor E. Sex differences in the association of physical function and cognitive function with life satisfaction in older age: The Rancho Bernardo study. Maturitas. 2016; 89:29-35. [DOI:10.1016/j.maturitas.2016.04.007]

[36] Wood V, Wylie M, Sheafor B. An analysis of a short self-report measure of life satisfaction: Correlation with rate judgment. Journal of Gerontology. 1969; 24(4):465-69. [DOI:10.1093/geronj/24.4.465] [PMID]

[37] Lawton M. The functional assessment of elderly people. Journal of the American Geriatrics Society. 1971; 19(6):465-81. [DOI:10.1111/j.1532-5415.1971.tb01206.x] [PMID]

[38] Alipour A, Agah Heris M. [Reliability and validity of the oxford happiness inventory among Iranians (Persian)]. Journal of Iranian Psychologists. Summer. 2007; 3(12):287-98.

[39] Vatankhah M, Hamid N, Mehrabizadeh Honarmad M. [The efficacy of quality of life group training on happiness and vitality of aged men (Persian)]. The Journal of Urmia University of Medical Sciences. 2013; 24(10):800-11.

[40] Almasi M, Mahmoudiani S, Jafari M. Factors associated with feelings of happiness among the elderly. Iran Journal of Nursing. 2015; 27(92):23-32. [DOI:10.29252/ijn.27.92.23]

[41] Keykhosravi Z, Hosieni S, Rezaei A. A comparison of old men and women's social support, life satisfaction, happiness and depression. Journal of Woman and Society. 2012; 2(8):161-43.

[42] Borg C, Hallberg I, Blomqvist K. Life satisfaction among older people $(65+)$ with reduced self-care capacity: The relationship to social, health and financial aspects. Journal of Clinical Nursing. 2006; 15(5):607-18. [DOI:10.1111/j.13652702.2006.01375.x] [PMID]

[43] Madah SB. [The status of social and leisure time activities in the elderly residing in Iran and Sweden (Persian)]. Iranian Journal of Ageing. 2008; 3(2):597-606

[44] McAuley E, Blissmer B, Marquez DX, Jerome GJ, Kramer AF, Katula J. Social relations, physical activity, and well-being in older adults. Preventive Medicine. 2000; 31(5):608-17. [DOI:10.1006/ pmed.2000.0740] [PMID]

[45] Meyers DG, Diener EE. Who is happy. Psychological Science. 2004; 6(1):10-9. [DOI:10.1111/j.1467-9280.1995.tb00298.x]

[46] Lu L. Social support, reciprocity, and well-being. The Journal of Social Psychology. 1997; 137(5):618-28. [DOI:10.1080/00224549709595483] [PMID]

[47] Lachiyani F, Mohammadi Arya A, RobatJazee ES, Tabatabaee SSh, Hedayati M, Rostaee A. [The efficacy of stress inoculation training upon the happiness rate and life's quality of old persons (Persian)]. Journal of Sabzevar University of Medical Sciences. 2013; 20(2):184-93. 